\title{
Time-Frequency Based Channel Estimation for High-Mobility OFDM Systems-Part I: MIMO Case
}

\author{
Erol Önen, ${ }^{1}$ Aydin Akan (EURASIP Member), ${ }^{1}$ and Luis F. Chaparro (EURASIP Member) ${ }^{2}$ \\ ${ }^{1}$ Department of Electrical and Electronics Engineering, Istanbul University, Avcilar, 34320 Istanbul, Turkey \\ ${ }^{2}$ Department of Electrical and Computer Engineering, University of Pittsburgh, Pittsburgh, PA 15261, USA \\ Correspondence should be addressed to Aydın Akan, akan@istanbul.edu.tr
}

Received 1 February 2010; Accepted 14 May 2010

Academic Editor: Lutfiye Durak

Copyright ( $\odot 2010$ Erol Önen et al. This is an open access article distributed under the Creative Commons Attribution License, which permits unrestricted use, distribution, and reproduction in any medium, provided the original work is properly cited.

\begin{abstract}
Multiple-input multiple-output (MIMO) systems hold the potential to drastically improve the spectral efficiency and link reliability in future wireless communications systems. A particularly promising candidate for next-generation fixed and mobile wireless systems is the combination of MIMO technology with Orthogonal Frequency Division Multiplexing (OFDM). OFDM has become the standard method because of its advantages over single carrier modulation schemes on multipath, frequency selective fading channels. Doppler frequency shifts are expected in fast-moving environments, causing the channel to vary in time, that degrades the performance of OFDM systems. In this paper, we present a time-varying channel modeling and estimation method based on the Discrete Evolutionary Transform to obtain a complete characterization of MIMO-OFDM channels. Performance of the proposed method is evaluated and compared on different levels of channel noise and Doppler frequency shifts.
\end{abstract}

\section{Introduction}

The major challenges in future wireless communications systems are increased spectral efficiency and improved link reliability. The wireless channel constitutes a hostile propagation medium, which suffers from fading (caused by destructive addition of multipath components) and interference from other users. Diversity provides the receiver with several (ideally independent) replicas of the transmitted signal and is therefore a powerful means to combat fading and interference and thereby improve link reliability. Common forms of diversity are space-time diversity [1] and space-frequency diversity [2]. In recent years the use of spatial (or antenna) diversity has become very popular, which is mostly due to the fact that it can be provided without loss in spectral efficiency. Receive diversity, that is, the use of multiple antennas on the receiver side of a wireless link, is a well-studied subject [3]. Driven by mobile wireless applications, where it is difficult to deploy multiple antennas in the handset, the use of multiple antennas on the transmitter side combined with signal processing and coding has become known under the name of space-time coding [4] and is currently an active area of research. The use of multiple antennas at both ends of a wireless link (multiple-input multiple-output (MIMO) technology) has been demonstrated to have the potential of achieving extraordinary data rates [5]. The corresponding technology is known as spatial multiplexing [6] or BLAST [7] and yields an impressive increase in spectral efficiency. Most of the previous work in the area of MIMO wireless has been restricted to narrow-band systems. Besides spatial diversity broadband MIMO channels, however, offer to higher capacity and frequency diversity due to delay spread. Orthogonal frequency division multiplexing (OFDM) significantly reduces receiver complexity in wireless broadband systems. The use of MIMO technology in combination with OFDM, that is, MIMO-OFDM [6], therefore seems to be an attractive solution for future broadband wireless systems $[8,9]$. However, intercarrier interference (ICI) due to Doppler shifts, phase offset, local oscillator frequency shifts, and multi-path fading severely degrades the performance of OFDM systems [10]. Most of the channel estimation methods assume a linear timeinvariant model for the channel, which is not valid for the next-generation, fast-moving environments [11]. Recently 
a time-frequency varying MIMO-OFDM channel estimation approach is presented where discrete prolate spheroidal sequences are used to obtain a robust time-varying channel estimator that does not require any channel statistics [12]. A time-varying model of the channel can be obtained by employing time-frequency representation methods. Here we present a time-varying MIMO-OFDM channel estimation based on the discrete evolutionary representation of the channel output. The Discrete Evolutionary Transform (DET) [13] provides a time-frequency representation of the received signal by means of which the spreading function of the multipath, fading, and frequency selective channel can be modeled and estimated.

The rest of the paper is organized as follows. In Section 2, we give a brief summary of the wireless parametric channel model used in our approach and the MIMOOFDM communication system. Section 3 presents timevarying modeling and estimation of MIMO-OFDM channels via DET. A time-frequency receiver is also given in Section 3 for the detection of data symbols using estimated channel parameters. In Section 4, we give some simulation results to illustrate the performance of our algorithm for different levels of channel noise and Doppler frequency-shifts and compare with other existing methods. Conclusions are drawn in Section 5.

\section{MIMO-OFDM System Model}

In this section we give a brief introduction to the timevarying, parametric communication channel model used in our work and the MIMO-OFDM signal model.

2.1. Parametric Channel Model. In wireless communications, the multi-path, fading channel with Doppler frequencyshifts may be modeled as a linear time-varying system with the following impulse response [14-16]:

$$
h(t, \tau)=\sum_{p=0}^{L_{p}-1} \gamma_{p}(t) \delta\left(\tau-\tau_{p}\right)
$$

where $\gamma_{p}(t)$ are independent Gaussian processes with zero mean and $\sigma_{p}^{2}$ variance, $\left\{\tau_{p}\right\}$ are delay profiles describing the channel dispersion with $\tau_{\max }$ as the maximum delay, and $L_{p}$ is the total number of paths. The variance $\sigma_{p}^{2}$ is a measure of the average signal power received at path $p$, characterized by the relative attenuation of that path, $\alpha_{p}$. Assuming that the sampling frequency is high enough, so the delays are integer multiples of the sampling interval, a discrete-time channel model can be obtained as $[15,17]$

$$
h(m, \ell)=\sum_{p=0}^{L_{p}-1} \alpha_{p} e^{j \psi_{p} m} \delta\left(\ell-N_{p}\right)
$$

where $m$ is the time index, $\psi_{p}$ represents the Doppler frequency-shift, $\alpha_{p}$ is the relative attenuation, and $N_{p}$ is the delay in path $p$. The Doppler frequency shift $\psi$, on the carrier frequency $\omega_{c}$, is caused by an object with radial velocity $v$ and can be approximated by [15]

$$
\psi \cong \frac{v}{c} \omega_{c}
$$

where $c$ is the speed of light in the transmission medium. In the new generation wireless mobile communication systems, with fast moving objects and high carrier frequencies, Doppler frequency-shifts become significant and have to be taken into consideration. The channel parameters cannot be easily estimated from the impulse response; however the estimation problem can be solved in the time-frequency domain by means of the so called spreading function.

The spreading function is related to the generalized transfer function and the bifrequency function. The generalized transfer function of the linear, time-varying channel is obtained by taking the DFT of $h(m, \ell)$ with respect to $\ell$, that is,

$$
H\left(m, \omega_{k}\right)=\sum_{p=0}^{L_{p}-1} \alpha_{p} e^{j \psi_{p} m} e^{-j \omega_{k} N_{p}},
$$

where $\omega_{k}=(2 \pi / K) k, k=0,1, \ldots, K-1$. Now, the channel bi-frequency function is found by computing the DFT of $H\left(m, \omega_{k}\right)$ with respect to time variable, $m$ :

$$
B\left(\Omega_{s}, \omega_{k}\right)=\sum_{p=0}^{L_{p}-1} \alpha_{p} e^{-j \omega_{k} N_{p}} \delta\left(\Omega_{s}-\psi_{p}\right),
$$

where $\Omega_{s}=(2 \pi / N) s, s=0,1, \ldots, N-1$. Calculating the inverse DFT of $B\left(\Omega_{s}, \omega_{k}\right)$ with respect to $\omega_{k}$ (or by taking the DFT of $h(m, \ell)$ in (2) with respect to $m)$, we have the spreading function of the channel as

$$
S\left(\Omega_{s}, k\right)=\sum_{p=0}^{L_{p}-1} \alpha_{p} \delta\left(\Omega_{s}-\psi_{p}\right) \delta\left(k-N_{p}\right)
$$

provided that the Doppler frequency shifts are integer multiples of the frequency sampling interval $\Omega_{s} . S\left(\Omega_{s}, k\right)$ displays peaks located at the time-frequency positions determined by the delays and the corresponding Doppler frequencies, with $\alpha_{p}$ as their amplitudes [17]. In our approach, we extract this information from the spreading function of the received signal and then detect the transmitted data symbol.

2.2. MIMO-OFDM Signal Model. In an OFDM communication system, the available bandwidth $B_{d}$ is divided into $K$ subchannels. The input data is also divided into $K$-bit parallel bit streams and then mapped onto some transmit symbols $X_{n, k}$ drawn from an arbitrary constellation points where $n$ is the time index, and $k=0,1, \ldots, K-1$, denotes the frequency or subcarrier index.

Some pilot symbols are inserted at some preassigned positions $\left(n^{\prime}, k^{\prime}\right)$, known to the receiver: $\left(n^{\prime}, k^{\prime}\right) \in P=$ $\left\{\left(n^{\prime}, k^{\prime}\right) \mid n^{\prime} \in Z, k^{\prime}=i S+\left(n^{\prime} \bmod (S)\right), i \in[0, P-1]\right\}$, where $P$ is the number of pilots, and the integer $S=K / P$ is the distance between adjacent pilots in an OFDM symbol 
[10]. The $n$th OFDM symbol $s_{n}(m)$ is obtained by taking $K$ point inverse DFT and then adding a cyclic prefix $(\mathrm{CP})$ of length $L_{\mathrm{CP}}$ where $L_{\mathrm{CP}}$ is chosen such that $L \leq L_{\mathrm{CP}}+1$, and $L$ is the time-support of the channel impulse response. This is done to mitigate the effects of intersymbol interference (ISI) caused by the channel dispersion in time.

A MIMO-OFDM system with $N_{\text {tx }}$ transmit and $N_{\text {rx }}$ receive antennas is depicted in Figure 1. The incoming bits are modulated to form $X_{n, k}^{(i)}$, where $i$ is the transmit antenna index. After parallel-to-serial $(\mathrm{P} / \mathrm{S})$ conversion, the signal transmitted by the $i$ th antenna becomes

$$
s_{n}^{(i)}(m)=\frac{1}{\sqrt{K}} \sum_{k=0}^{K-1} X_{n, k}^{(i)} e^{j \omega_{k} m},
$$

where $m=-L_{\mathrm{CP}},-L_{\mathrm{CP}}+1, \ldots, 0, \ldots, K-1, \omega_{k}=(2 \pi / K) k$, and each OFDM symbol is $N=K+L_{\mathrm{CP}}$ sample long. The channel output suffers from multi-path propagation, fading and Doppler frequency shifts:

$$
\begin{aligned}
y_{n}^{(j)}(m) & =\sum_{i=1}^{N_{\mathrm{tx}}}\left(\sum_{\ell=0}^{L_{p}^{(i, j)}-1} h^{(i, j)}(m, \ell) s_{n}^{(i)}(m-\ell)\right) \\
& =\sum_{i=1}^{N_{\mathrm{tx}}}\left(\sum_{p=0}^{L_{p}^{(i, j)}-1} \alpha_{p}^{(i, j)} e^{j \psi_{p}^{(i, j)} m} s_{n}^{(i)}\left(m-N_{p}^{(i, j)}\right)\right) \\
& =\sum_{i=1}^{N_{\mathrm{tx}}}\left(\frac{1}{\sqrt{K}} \sum_{k=0}^{K-1} X_{n, k}^{(i)} \sum_{p=0}^{L_{p}^{(i, j)}-1} \alpha_{p}^{(i, j)} e^{j \psi_{p}^{(i, j)}} e^{j \omega_{k}\left(m-N_{p}^{(i, j)}\right)}\right) \\
& =\sum_{i=1}^{N_{\mathrm{tx}}}\left(\frac{1}{\sqrt{K}} \sum_{k=0}^{K-1} H_{n}^{(i, j)}\left(m, \omega_{k}\right) e^{j \omega_{k} m} X_{n, k}^{(i)}\right),
\end{aligned}
$$

where $(\cdot)^{(j)}$ denotes the $j$ th receiver. $(\cdot)^{(i, j)}$ is indexing for the wireless time-varying channel between the $i$ th transmitter and the $j$ th receiver antennas. The transmit signal is also corrupted by additive white Gaussian noise $\eta^{(j)}(m)$ over the channel. The received signal for the $n$th frame can then be written as $r_{n}^{(j)}(m)=y_{n}^{(j)}(m)+\eta_{n}^{(j)}(m)$. The receiver discards the Cyclic Prefix and demodulates the signal using a $K$-point DFT as

$$
\begin{aligned}
& R_{n, k}^{(j)}= \frac{1}{\sqrt{K}} \sum_{m=0}^{K-1}\left[y_{n}^{(j)}(m)+\eta_{n}^{(j)}(m)\right] e^{-j \omega_{k} m} \\
&=\sum_{i=1}^{N_{\mathrm{tx}}}\left(\frac{1}{\sqrt{K}} \sum_{s=0}^{K-1} X_{n, s}^{(i)} \sum_{p=0}^{L_{p}^{(i, j)}-1} \alpha_{p}^{(i, j)} e^{j \omega_{s}\left(m-N_{p}^{(i, j)}\right)}\right. \\
&\left.\quad \times \sum_{m=0}^{K-1} e^{j \psi_{p}^{(i, j)} m} e^{j\left(\omega_{s}-\omega_{k}\right) m}+N_{n, k}^{(j)}\right)
\end{aligned}
$$

where $N_{n, k}^{(j)}$ is the Fourier transform of the channel noise at the $j$ th receiver. If the Doppler effects in all the channel paths are negligible, $\psi_{p}^{(i, j)}=0$, for all $i, j$, then the channel is almost time-invariant within one OFDM symbol. In that case, above equation becomes

$$
\begin{aligned}
R_{n, k}^{(j)} & =\sum_{i=1}^{N_{\mathrm{tx}}} X_{n, k}^{(i)} \sum_{p=0}^{L_{p}^{(i, j)}-1} \alpha_{p}^{(i, j)} e^{-j \omega_{k} N_{p}^{(i, j)}}+N_{n, k}^{(j)} \\
& =\sum_{i=1}^{N_{\mathrm{tx}}} H_{n, k}^{(i, j)} X_{n, k}^{(i)}+N_{n, k}^{(j)},
\end{aligned}
$$

where $H_{n, k}^{(i, j)}$ is the frequency response of the channel between the $i$ th transmitter and $j$ th receiver antenna, and if there are large Doppler frequency shifts in the channel, then the timeinvariance assumption above is no longer valid. Here we consider modeling and estimation of the channels frequency responses $H_{n, k}^{(i, j)}$ and approach the problem from a timefrequency point of view [17].

\section{Time-Varying Channel Estimation for MIMO-OFDM Systems}

In this section we present a time-frequency procedure to characterize time-varying MIMO-OFDM channels. We also propose a time-frequency receiver that uses the estimated channel fading, delay, and Doppler parameters to recover the transmitted symbols. In the following, we briefly present the Discrete Evolutionary Transform (DET) as a tool for the time-frequency representation of time-varying MIMOOFDM channels.

3.1. The Discrete Evolutionary Transform. Wold-Cramer representation [18] of a nonstationary random signal $\gamma(n)$ can be expressed as an infinite sum of sinusoids with random and time-varying amplitudes and phases, or

$$
\gamma(n)=\int_{-\pi}^{\pi} \Gamma(n, \omega) e^{j \omega n} d Z(\omega),
$$

where $Z(\omega)$ is considered a random process with orthogonal increments. This is a generalization of the spectral representation of stationary processes. Priestley's evolutionary spectrum [18, 19] of $\gamma(n)$ is given as the magnitude square of the evolutionary kernel $\Gamma(n, \omega)$. Analogous to the above Wold-Cramer representation, a discrete, timefrequency representation for a deterministic signal $x(n)$ with a time-dependent spectrum is possible $[13,20]$ :

$$
x(n)=\sum_{k=0}^{K-1} X\left(n, \omega_{k}\right) e^{j \omega_{k} n}, \quad 0 \leq n \leq N-1,
$$

where $\omega_{k}=2 \pi k / K, K$ is the number of frequency samples, and $X\left(n, \omega_{k}\right)$ is a time-frequency evolutionary kernel. A similar representation can be given in terms of the corresponding bi-frequency kernel $X\left(\Omega_{s}, \omega_{k}\right)$ :

$$
x(n)=\sum_{k=0}^{K-1} \sum_{s=0}^{K-1} X\left(\Omega_{s}, \omega_{k}\right) e^{j\left(\omega_{k}+\Omega_{s}\right) n},
$$




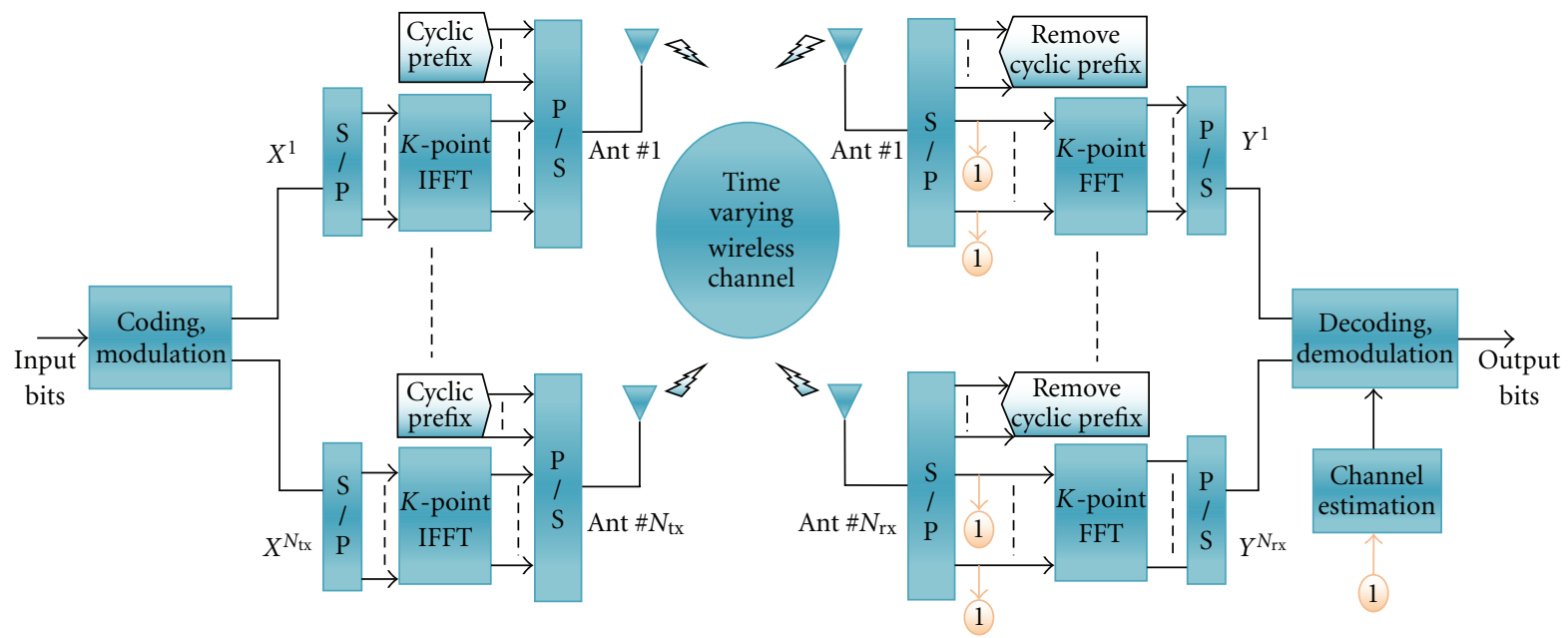

FIgure 1: MIMO-OFDM System Model.

where $\omega_{k}$ and $\Omega_{s}$ are discrete frequencies. Discrete evolutionary transformation (DET) is obtained by expressing the kernels $X\left(n, \omega_{k}\right)$ or $X\left(\Omega_{s}, \omega_{k}\right)$ by means of the signal [13]. Thus, for the representation in (12) the DET that provides the evolutionary kernel $X\left(n, \omega_{k}\right), 0 \leq k \leq K-1$, is given by

$$
X\left(n, \omega_{k}\right)=\sum_{\ell=0}^{N-1} x(\ell) \mathrm{w}_{k}(n, \ell) e^{-j \omega_{k} \ell}
$$

where $\mathrm{w}_{k}(n, \ell)$ is, in general, a time- and frequencydependent window.

The DET can be seen as a generalization of the shorttime Fourier transform, where the windows are constant. The windows $\mathrm{w}_{k}(n, \ell)$ can be obtained from either the Gabor representation that uses nonorthogonal frames or the Malvar wavelet representation that uses orthogonal systems. Details of how the windows can be obtained for the Gabor and Malvar representations are given in [13]. However, for the representation of multipath wireless channel outputs, we consider windows that are adapted to the Doppler frequencies of the channel.

3.2. MIMO-OFDM Channel Estimation by Using DET. We will now consider the computation of the spreading function by means of the evolutionary transformation of the received signal. The output of the channel, after discarding the cyclic prefix, for the $n$th OFDM symbol can be written using (8) as

$$
\begin{aligned}
y_{n}^{(j)}(m) & =\sum_{i=1}^{N_{\mathrm{xx}}}\left(\frac{1}{\sqrt{K}} \sum_{p=0}^{L_{p}^{(i, j)}-1} \sum_{k=0}^{K-1} \alpha_{p}^{(i, j)} e^{j \psi_{p}^{(i, j)} m} e^{j \omega_{k}\left(m-N_{p}^{(i, j)}\right)} X_{n, k}^{(i)}\right) \\
& =\frac{1}{\sqrt{K}} \sum_{p=0}^{L_{p}^{(i, j)}-1} H_{n}^{(i, j)}\left(m, \omega_{k}\right) e^{j \omega_{k} m} X_{n, k}^{(i)},
\end{aligned}
$$

where we ignore the additive channel noise $\eta^{(j)}(m)$ for simplicity. The equation above can be rewritten in matrix form as

$$
\mathbf{y}^{(\mathbf{j})}=\mathbf{H}^{(\mathbf{j})} \mathbf{x}
$$

where

$$
\begin{gathered}
\mathbf{y}^{(j)}=\left[y_{n}^{(j)}(0), y_{n}^{(j)}(1), \ldots, y_{n}^{(j)}(K-1)\right]^{T} ; \\
\mathbf{x}=\left[\mathbf{x}^{(1)}, \mathbf{x}^{(2)}, \ldots, \mathbf{x}^{\left(N_{\mathrm{tx}}\right)}\right]^{T}, \\
\mathbf{x}^{(i)}=\left[X_{n, 0}^{(i)}, X_{n, 1}^{(i)}, \ldots, X_{n, K-1}^{(i)}\right] ; \\
\mathbf{H}^{(i, j)}=\left[a_{m, k}\right]_{K \times K}, \quad a_{m, k}=\frac{H_{n}^{(i, j)}\left(m, \omega_{k}\right) e^{j \omega_{k} m}}{\sqrt{K}} ; \\
\mathbf{H}^{(j)}=\left[\mathbf{H}^{(1, j)}, \mathbf{H}^{(2, j)}, \ldots, \mathbf{H}^{\left(N_{\mathrm{tx}}, j\right)}\right] .
\end{gathered}
$$

The input-output relation for the whole system results as

$$
\mathbf{y}=\mathbf{H x} \text {, }
$$

where

$$
\mathbf{H}=\left[\begin{array}{c}
\mathbf{H}^{(1)} \\
\mathbf{H}^{(2)} \\
\vdots \\
\mathbf{H}^{\left(N_{\mathrm{rx}}\right)}
\end{array}\right], \quad \mathbf{y}=\left[\begin{array}{c}
\mathbf{y}^{(1)} \\
\mathbf{y}^{(2)} \\
\vdots \\
\mathbf{y}^{\left(N_{\mathrm{rx}}\right)}
\end{array}\right] .
$$

If $\mathbf{H}$ is known and then input symbols can be estimated by the following relation,

$$
\widehat{\mathbf{x}}=\mathbf{H}^{-1} \mathbf{y}
$$

Now calculating the discrete evolutionary representation of $y_{n}^{(j)}(m)$, we get

$$
y_{n}^{(j)}(m)=\sum_{k=0}^{K-1} Y_{n}^{(j)}\left(m, \omega_{k}\right) e^{j \omega_{k} m} .
$$


Comparing the representations of $y_{n}^{(j)}(m)$ in (21) and (15), we get the kernel as

$$
Y_{n}^{(j)}\left(m, \omega_{k}\right)=\sum_{i=1}^{N_{\mathrm{tx}}}\left(\frac{1}{\sqrt{K}} H_{n}^{(i, j)}\left(m, \omega_{k}\right) X_{n, k}^{(i)}\right) .
$$

above relation is also valid at the preassigned pilot positions $k=k^{\prime}$ :

$$
\begin{aligned}
Y_{n}^{(j)}\left(m, \omega_{r}\right) & =Y_{n}^{\prime(j)}\left(m, \omega_{k^{\prime}}\right) \\
& =\sum_{i=1}^{N_{\mathrm{tx}}}\left(\frac{1}{\sqrt{K}} H_{n}^{(i, j)}\left(m, \omega_{k^{\prime}}\right) X_{n, k^{\prime}}^{(i)}\right),
\end{aligned}
$$

where $r=1,2, \ldots, P$ and $H_{n}^{(i, j)}\left(m, \omega_{k^{\prime}}\right)$ is a decimated version of the $H_{n}^{(i, j)}\left(m, \omega_{k}\right)$. Note that $P$ is again the number of pilots, and $S=K / P$ is the distance between adjacent pilots. Finally if the pilot symbols are chosen to be orthogonal to each other, the decimated frequency response of the channel between the $i$ th transmitter and the $j$ th receiver antennas may be obtained as

$$
H_{n}^{\prime(i, j)}\left(m, \omega_{r}\right)=\frac{\sqrt{K}}{\left\|X_{n, r}^{\prime(i)}\right\|}\left\langle Y_{n}^{\prime(j)}\left(m, \omega_{r}\right), X_{n, r}^{\prime(i)}\right\rangle .
$$

Taking the inverse DFT of $H_{n}{ }^{(i, j)}\left(m, \omega_{r}\right)$ with respect to $\omega_{r}$ and DFT with respect to $m$, we obtain the downsampled spreading function $S^{\prime}\left(\Omega_{s}, \ell\right)$,

$S_{n}^{\prime(i, j)}\left(\Omega_{s}, \ell\right)=\frac{1}{S} \sum_{p=0}^{L_{p}^{(i, j)}-1} \alpha_{p}^{(i, j)} \delta_{n}^{(i, j)}\left(\Omega_{s}-\psi_{p}^{(i, j)}\right) \delta_{n}^{(i, j)}\left(\frac{\ell-N_{p}^{(i, j)}}{S}\right)$.

By comparing $S_{n}^{(i, j)}\left(\Omega_{s}, \ell\right)$ and $S_{n}^{(i, j)}\left(\Omega_{s}, \ell\right)$, we observe that the channel parameters $\alpha_{p}^{(i, j)}, N_{p}^{(i, j)}$, and $\psi_{p}^{(i, j)}$ calculated from $S_{n}^{(i, j)}\left(\Omega_{s}, \ell\right)$ can also be estimated from the downsampled spreading function $S_{n}^{(i, j)}\left(\Omega_{s}, \ell\right)$.

In the following, we present a method to estimate the spreading function of the MIMO channel from the received signal. The time-frequency evolutionary kernel of the channel output in the $j$ th receiver is obtained as

$$
\begin{aligned}
Y_{n}^{(j)}\left(m, \omega_{k}\right)= & \sum_{\ell=0}^{K-1} y_{n}^{(j)}(\ell) \mathrm{w}_{k}(m, \ell) e^{-j \omega_{k} \ell} \\
= & \sum_{i=1}^{N_{\mathrm{tx}}}\left(\frac{1}{\sqrt{K}} \sum_{s=0}^{K-1} X_{n, \mathcal{S}}^{(i)} \sum_{p=0}^{L_{p}^{(i, j)}-1} \alpha_{p}^{(i, j)} e^{-\omega_{s} N_{p}^{(i, j)}}\right. \\
& \left.\quad \times \sum_{\ell=0}^{N-1} \mathrm{w}_{k}(m, \ell) e^{j\left(\psi_{p}^{(i, j)}+\omega_{s}-\omega_{k}\right) \ell}\right) .
\end{aligned}
$$

We consider windows of the form $\mathrm{w}_{r}(m, \ell)=e^{j \psi_{r}(m-\ell)}$, for $0 \leq \psi_{r} \leq \pi$ presented in [17] that depends on the Doppler frequency $\psi_{r}$. This window will give us the correct representation of $Y_{n}^{(j)}\left(m, \omega_{k}\right)$ only when $\psi_{r}=\psi_{p}^{(i, j)}$; in fact, using the window $\mathrm{w}_{r}(m, \ell)=e^{j \psi_{p}^{(i, j)}(m-\ell)}$, the above representation of $Y_{n}^{(j)}\left(m, \omega_{k}\right)$ becomes,

$$
Y_{n}^{(j)}\left(m, \omega_{k}\right)=\sum_{i=1}^{N_{\mathrm{tx}}} \sqrt{K} H_{n}^{(i, j)}\left(m, \omega_{k}\right) X_{n, k}^{(i)}
$$

which is the expected result multiplied by $K$. In our estimation procedure, we use windows $\mathrm{w}_{u}(m, \ell)=e^{j \omega_{u}(m-\ell)}$ where $\omega_{u}$ is chosen in a discrete set with certain increments, $\omega_{u}=\pi u / U, u=0,1,2, \ldots, U-1$. When $\omega_{u}$ coincides with one of the Doppler frequencies in the channel, the spreading function displays a large peak at the time-frequency position $\left(N_{p}^{(i, j)}, \psi_{p}^{(i, j)}\right)$, corresponding to delay and Doppler frequency of that transmission path, with magnitude proportional to attenuation $\alpha_{p}^{(i, j)}$. When $\omega_{u}$ does not coincide with any of the Doppler frequencies, the spreading function displays a random sequence of peaks spread over all possible delays. Then it is possible to determine a threshold that permits us to obtain the most significant peaks of the spreading function corresponding to possible delays and Doppler frequencies. In our experiments we observed that peaks having amplitudes larger than $65 \%$ of the maximum peak are due to an actual transmission; otherwise they are considered as noise. Thus, by searching in the possible Doppler frequency range, we are able to estimate all the parameters of a multi-path, fading, and time-varying MIMO-OFDM channel via the spreading function of the channel.

According to (27), we need the input pilot symbols $X_{n, k^{\prime}}$ to estimate the channel frequency response. Here we consider simple, uniform pilot patterns; however improved patterns may be employed as well [11].

3.3. Time-Frequency Receiver. After estimating the spreading function and the corresponding frequency response $H_{n}^{(i, j)}\left(m, \omega_{k}\right)$ of the channel, data symbols $X_{n, k}^{(i)}$ can be detected using a time-frequency receiver given in (20). On the other hand, the channel output in (9) can be rewritten as

$$
\begin{aligned}
R_{n, k}^{(j)} & =\sum_{i=1}^{N_{\mathrm{tx}}}\left(\frac{1}{K} \sum_{s=0}^{K-1}\left\{\sum_{m=0}^{K-1} H_{n}^{(i, j)}\left(m, \omega_{k}\right) e^{j\left(\omega_{s}-\omega_{k}\right) m}\right\} X_{n, s}^{(i)}\right)+N_{n, k}^{(j)} \\
& =\frac{1}{K} \sum_{s=0}^{K-1} B_{n}^{(i, j)}\left(\omega_{k}-\omega_{s}, \omega_{s}\right) X_{n, s}+N_{n, k} \\
& =\sum_{i=1}^{N_{\mathrm{tx}}}\left(\frac{1}{K} \sum_{s=0}^{K-1} B_{n}^{(i, j)}\left(\omega_{k}-\omega_{s}, \omega_{s}\right) X_{n, s}^{(i)}\right)+N_{n, k}^{(j)},
\end{aligned}
$$

where $B_{n}^{(i, j)}\left(\Omega_{s}, \omega_{k}\right)$ is the bi-frequency function of the channel during $n$th OFDM symbol, and the above equation indicates a circular convolution with the data symbols. Based 
on aforementioned equality it is possible to write the MIMOOFDM system consisting of $N_{\mathrm{tx}}$ transmitter and $N_{\mathrm{rx}}$ receiver antennas in a matrix form as

$$
\left[\begin{array}{c}
\mathbf{r}^{(1)} \\
\vdots \\
\mathbf{r}^{\left(N_{\mathrm{rx}}\right)}
\end{array}\right]=\left[\begin{array}{ccc}
\mathbf{B}^{(1,1)} & \cdots & \mathbf{B}^{\left(N_{\mathrm{tx}}, 1\right)} \\
\vdots & \cdots & \vdots \\
\mathbf{B}^{\left(N_{\mathrm{rx}}, 1\right)} & \cdots & \mathbf{B}^{\left(N_{\mathrm{tx}}, N_{\mathrm{rx}}\right)}
\end{array}\right]\left[\begin{array}{c}
\mathbf{x}^{(1)} \\
\vdots \\
\mathbf{x}^{\left(N_{\mathrm{tx}}\right)}
\end{array}\right]+\left[\begin{array}{c}
\mathbf{z}^{(1)} \\
\vdots \\
\mathbf{z}^{\left(N_{\mathrm{rx}}\right)}
\end{array}\right]
$$

or

$$
\mathbf{r}=\mathbf{B x}+\mathbf{z}
$$

where $\mathbf{B}^{(i, j)}=\left[b_{s, k}\right]_{K \times K}=B_{n}^{(i, j)}\left(\omega_{k}-\omega_{s}, \omega_{s}\right)$ is a $K \times$ $K$ matrix; $\mathbf{r}, \mathbf{x}$, and $\mathbf{z}$ are $K \times 1$ vectors defined by $\mathbf{r}^{(j)}=\left[R_{n, 1}^{(j)}, R_{n, 2}^{(j)}, \ldots, R_{n, K}^{(j)}\right]^{T}, \mathbf{x}^{(j)}=\left[X_{n, 1}^{(j)}, X_{n, 2}^{(j)}, \ldots, X_{n, K}^{(j)}\right]^{T}$, and $\mathbf{z}^{(j)}=\left[N_{n, 1}^{(j)}, N_{n, 2}^{(j)}, \ldots, N_{n, K}^{(j)}\right]^{T}$, respectively. Finally, data symbols $X_{n, k}^{(j)}$ can be estimated by using a simple timefrequency receiver: $\widehat{\mathbf{x}}=\mathbf{B}^{-1} \mathbf{r}$.

The exhaustive search for the channel Doppler frequencies may seem to increase the computational cost of the proposed method. However, considering the carrier frequencies and maximum possible velocities in the environment, Doppler frequencies lie in a certain band which can be easily covered by the algorithm. Furthermore, our channel estimation approach does not require any a priori information on the statistics of the channel as in the case of many other channel estimation methods [11].

In the following, we demonstrate time-varying MIMOOFDM channel estimation performance of our timefrequency-based approach by means of examples.

\section{Simulations}

In the experiments, a 2-input, 2-output MIMO-OFDM system is considered, and the wireless channels are simulated randomly; that is, the number of paths, $1 \leq L_{p} \leq 5$, the delays, $0 \leq N_{p} \leq L_{\mathrm{CP}}-1$, and the Doppler frequency shift $0 \leq \psi_{p} \leq \psi_{\max }\left(p=0,1, \ldots, L_{p}-1\right)$ of each path are picked randomly. Input data is QPSK coded and modulated onto $K=128$ subcarriers, 16 of which are assigned to pilot symbols. The Signal-to-Noise Ratio (SNR) of the channel noise is changed between 5 and $35 \mathrm{~dB}$, for two different values of the maximum Doppler frequency. Figure 2 depicts an example of the estimated spreading function for a $2 \times 2$ MIMO system, during one OFDM symbol. In our simulations, we use Doppler frequencies normalized by the subcarrier spacing [21]:

$$
f_{D}=\frac{v f_{c}}{c} T_{s} N
$$

The performance of our channel estimation method is investigated and compared with that of a recently proposed time-frequency varying MIMO-OFDM channel estimation approach [12]. This method applies the discrete prolate spheroidal sequences to obtain a robust time-varying channel estimator that does not require any channel statistics, similar to our proposed method.

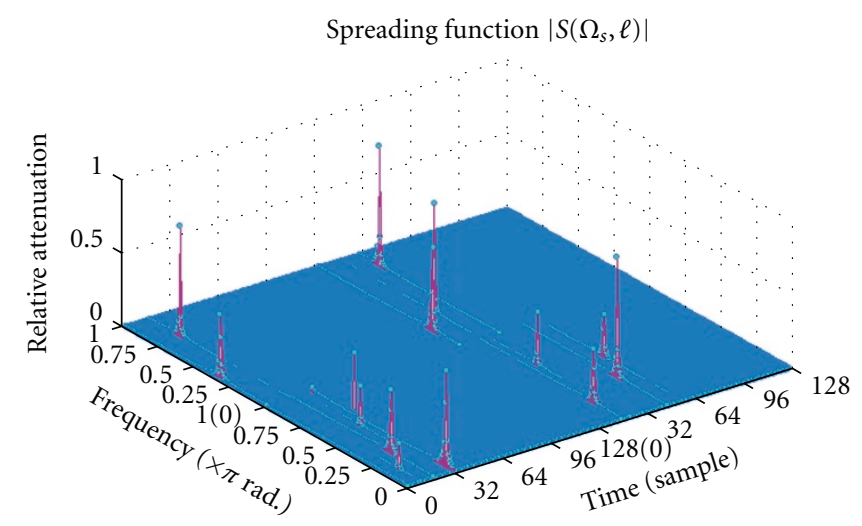

FIGURE 2: An example of estimated spreading function for the $2 \times 2$ MIMO-OFDM system.

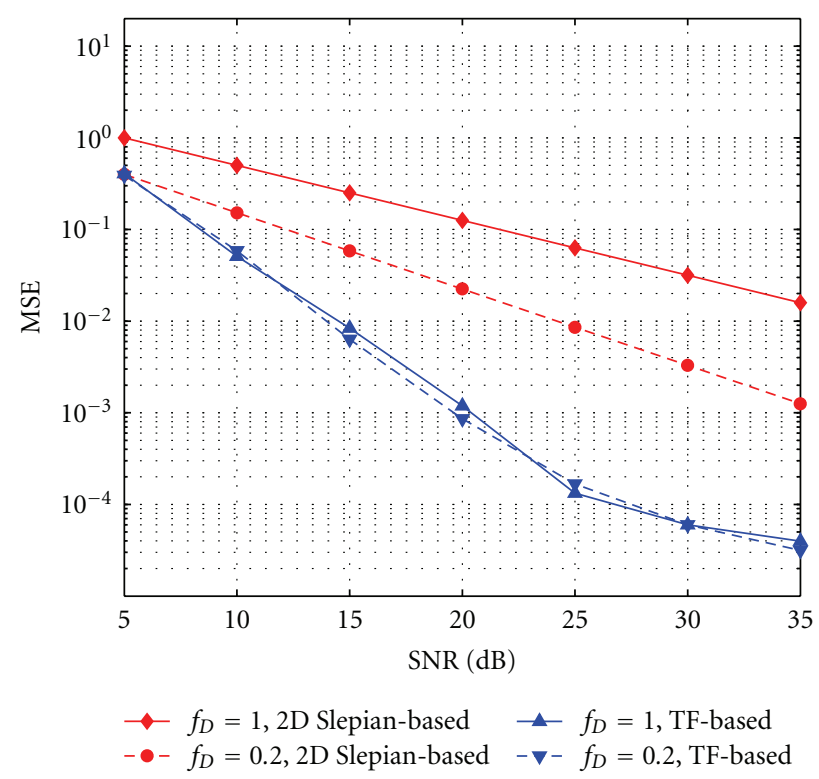

FIGURE 3: MSE for proposed channel estimation and the method presented in [12] for $2 \times 2$ MIMO-OFDM system for $f_{D}=0.2$ and $f_{D}=1$.

We show the mean square error (MSE) of our channel estimation approach and the 2D Slepian-based twodimensional channel estimation method [12] for different channel noise levels and for normalized Doppler frequencies $f_{D}=0.2$ and $f_{D}=1$ in Figure 3 . As seen from the graphs, our method outperforms the Slepian-based approach in terms of both estimation error and robustness against increased Doppler frequencies. Note that our method is capable of estimating and compensating for large Doppler shifts yielding a similar MSE for both $f_{D}=0.2$ and $f_{D}=1$.

We then investigate the bit error rate (BER) versus SNR of the channel noise performance of both channel estimation approaches for different numbers of the pilot symbols in one OFDM block, $P=\{8,16,32\}$ and show the results in Figure 4. Notice that for a fixed number of pilots, proposed TF-based approach achieves the same BER with 


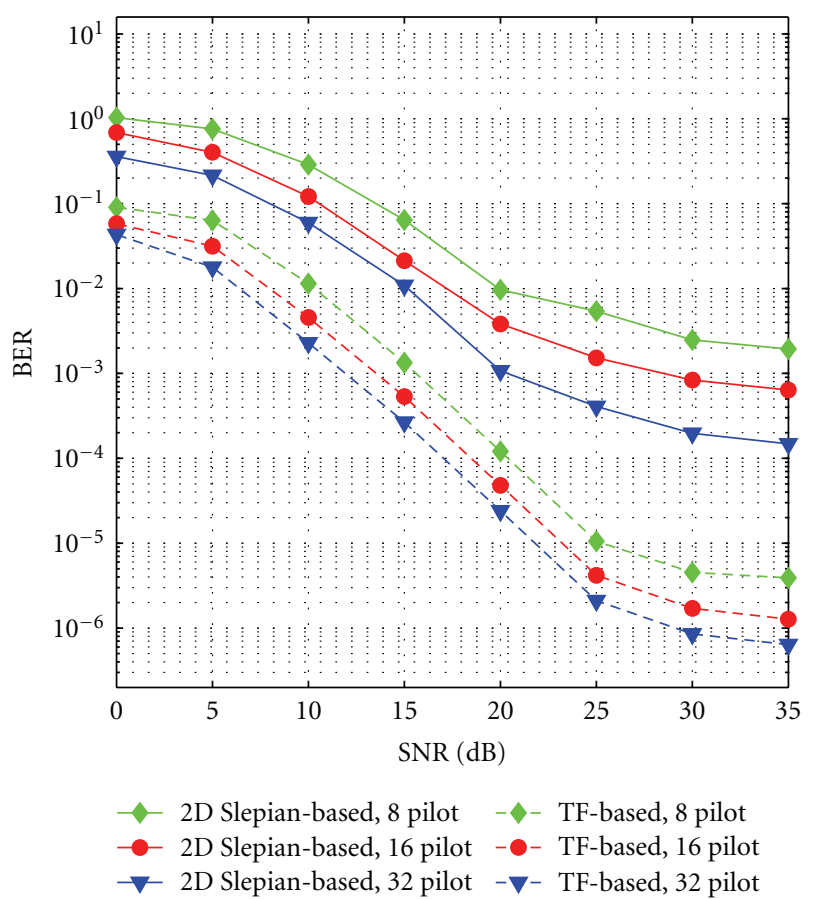

FIgURE 4: BER versus SNR performance of the TF-based and 2D Slepian-based method presented in [12] for $2 \times 2$ MIMO-OFDM system for 8,16 , and 32 pilots.

about $15 \mathrm{~dB}$ less SNR than the Slepian-based method. Note also that increasing the number of pilots improves the BER performance in both methods.

Finally the effect of the number of channel propagation paths is investigated. BER is calculated for both TF and Slepian-based channel estimation approaches by changing the number of paths from 3 to 25 and for $P=8$ and 16 pilots. Results given in Figure 5 show that for the same number of paths, proposed TF-based approach achieves approximately 100 times less BER than the 2D Slepian-based channel estimation method. Also notice that the BER performance goes down rapidly for the number of paths larger than 12.

\section{Conclusions}

In this work, we present a time-varying estimation of MIMO-OFDM channels for high-mobility communication systems by means of discrete evolutionary transform. The main advantage of the proposed method is that it does not assume any statistics on the communication channel. The parametric channel model used in this approach allows us to obtain a two-dimensional representation for the channel and estimate its parameters from the spreading function. We observe that the method is robust against large variations on the channel frequency response, that is, fast fading. Simulations show that our time-frequency-based method has considerably better channel estimation and bit error performance compared to a similar time-frequency varying channel estimation approach [12].

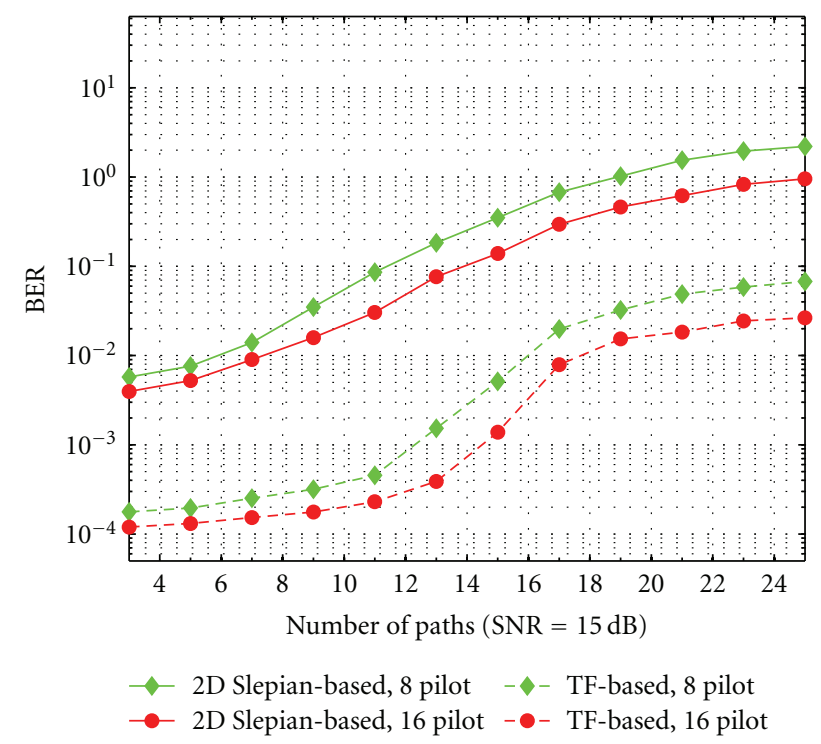

FIGURE 5: BER versus the number of channel paths for the TF-based channel estimation and the method presented in [12] for 8 and 16 pilots and $15 \mathrm{~dB}$ SNR.

\section{Acknowledgments}

This work was partially supported by The Research Fund of The University of Istanbul, Project nos. 6904, 3898, and 4382 .

\section{References}

[1] V. Tarokh, N. Seshadri, and A. R. Calderbank, "Space-time codes for high data rate wireless communication: performance criterion and code construction," IEEE Transactions on Information Theory, vol. 44, no. 2, pp. 744-765, 1998.

[2] H. A. Cirpan, E. Panayirci, and H. Doğan, "Nondata-aided channel estimation for OFDM systems with space-frequency transmit diversity," IEEE Transactions on Vehicular Technology, vol. 55, no. 2, pp. 449-457, 2006.

[3] A. Shah and A. M. Haimovich, "Performance analysis of optimum combining in wireless communications with Rayleigh fading and cochannel interference," IEEE Transactions on Communications, vol. 46, no. 4, pp. 473-479, 1998.

[4] S. M. Alamouti, "A simple transmit diversity technique for wireless communications," IEEE Journal on Selected Areas in Communications, vol. 16, no. 8, pp. 1451-1458, 1998.

[5] A. J. Paulraj and T. Kailath, "Increasing capacity in wireless broadcast systems using distributed transmission/directional reception,” US patent no. 5,345,599, 1994.

[6] H. Bölcskei, D. Gesbert, and A. J. Paulraj, "On the capacity of OFDM-based spatial multiplexing systems," IEEE Transactions on Communications, vol. 50, no. 2, pp. 225-234, 2002.

[7] G. J. Foschini and M. J. Gans, "On limits of wireless communications in a fading environment when using multiple antennas," Wireless Personal Communications, vol. 6, no. 3, pp. 311-335, 1998.

[8] A. Goldsmith, Wireless Communications, Cambridge University Press, New York, NY, USA, 2005. 
[9] T. Zemen, C. F. Mecklenbräuker, J. Wehinger, and R. R. Müller, "Iterative joint time-variant channel estimation and multiuser detection for MC-CDMA," IEEE Transactions on Wireless Communications, vol. 5, no. 6, pp. 1469-1478, 2006.

[10] G. L. Stüber, J. R. Barry, S. W. Mclaughlin, Y. E. Li, M. A. Ingram, and T. G. Pratt, "Broadband MIMO-OFDM wireless communications," Proceedings of the IEEE, vol. 92, no. 2, pp. 271-293, 2004.

[11] S. G. Kang, Y. M. Ha, and E. K. Joo, "A comparative investigation on channel estimation algorithms for OFDM in mobile communications," IEEE Transactions on Broadcasting, vol. 49, no. 2, pp. 142-149, 2003.

[12] P. S. Rossi and R. R. Müller, "Slepian-based two-dimensional estimation of time-frequency variant MIMO-OFDM channels," IEEE Signal Processing Letters, vol. 15, pp. 21-24, 2008.

[13] R. Suleesathira, L. F. Chaparro, and A. Akan, "Discrete evolutionary transform for time-frequency signal analysis," Journal of the Franklin Institute, vol. 337, no. 4, pp. 347-364, 2000.

[14] P. A. Bello, "Characterization of randomly time-variant linear channels," IEEE Transactions on Communications, vol. 11, no. 4, pp. 360-393, 1963.

[15] M. D. Hahm, Z. I. Mitrovski, and E. L. Titlebaum, "Deconvolution in the presence of Doppler with application to specular multipath parameter estimation," IEEE Transactions on Signal Processing, vol. 45, no. 9, pp. 2203-2219, 1997.

[16] F. Shu, J. Lee, L.-N. Wu, and G.-L. Zhao, "Time-frequency channel estimation for digital amplitude modulation broadcasting systems based on OFDM," IEE Proceedings on Communications, vol. 150, no. 4, pp. 259-264, 2003.

[17] A. Akan and L. F. Chaparro, "Modeling and estimation of wireless OFDM channels by using time-frequency analysis," Circuits, Systems, and Signal Processing, vol. 25, no. 3, pp. 389403, 2006.

[18] M. B. Priestley, Non-Linear and Non-Stationary Time Series Analysis, Academic Press, London, UK, 1988.

[19] G. Melard and A. H. Schutter, "Contributions to evolutionary spectral theory," Journal of Time Series Analysis, vol. 10, pp. 41-63, 1989.

[20] A. Akan and L. F. Chaparro, "Multi-window Gabor expansion for evolutionary spectral analysis," Signal Processing, vol. 63, no. 3, pp. 249-262, 1997.

[21] Z. Tang, R. C. Cannizzaro, G. Leus, and P. Banelli, "Pilotassisted time-varying channel estimation for OFDM systems," IEEE Transactions on Signal Processing, vol. 55, no. 5, pp. 2226$2238,2007$. 